\title{
MOVING AWAY FROM THE BANKING MODEL OF DENTAL EDUCATION
}

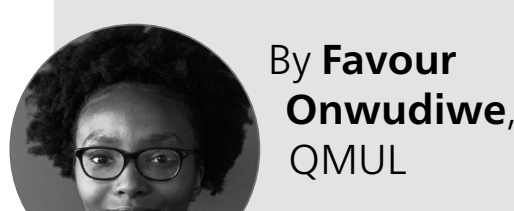

The Banking Model of Education is a concept in the 1968 book 'Pedagogy of the Oppressed' written by Paulo Freire. The 'banking' model of education is a style of learning and teaching in which students essentially store the knowledge given to them by the teacher. ${ }^{1}$

The primary responsibility of students in education in this setting is to remember and accurately recall the information. In the learning process, students do not engage actively. They simply absorb the information and regurgitate it. The universe is perceived as stagnant and unchangeable in this style of approach, and students are expected to fit into it as it is. ${ }^{1}$ Based on Paulo Freire's knowledge, this article discusses the problematic results of this approach and Freire's alternative teaching styles.

\section{How this applies to dentistry}

As far as dental education is concerned, tutors speak of diseases affecting patients as if they were mechanical, stagnant, compartmentalised, and predictable. Or illustrate typical dental diseases like tooth pain as completely alien to the human experience. ${ }^{1}$ Dental tutors are also charged with 'filling' the students with information of illnesses, totally disconnected from reality, isolated from nuanced experiences of life that produce signs and symptoms which appear differently in various classes of individuals, making diagnosis difficult.

The banking model of education also reduces one's ability to develop critical consciousness. It imposes a passive role on students who tend simply to adapt to the world as it is without any critical thinking. ${ }^{1}$

\section{'As far as dental education is concerned, tutors speak of diseases affecting patients as if they were mechanical, stagnant, compartmentalised, and predictable.'}

In evidence-based dentistry, for example, students are unconsciously taught that quantitative data is the gold standard, without questioning the reasoning behind it and the impact of discipline in dentistry.

In another example, the banking approach to health education would never recommend to students to critically consider reality but only to memorise and regurgitate facts. ${ }^{1}$ With challenges such as excessive sugar consumption, students regurgitate statements such as 'too much sugar can lead to tooth decay' without proper application of social determinants of tooth decay.

\section{A way forward}

Although some advantages of the banking model have a place in dentistry, i.e., the reproduction of time-tested principles and direct training in clinical skills laboratories, placing great importance on this model in dental education can create generations of dentists who lack imagination and critical thought. $^{2}$

Dentistry has been moving away from this traditional model by adding more elements of problem-based learning, less lecture-based learning and early clinical exposure. I believe there are still areas of improvement. Freire proposes a stronger emphasis on problem-posing teaching where teachers do not impart information to students rather raise questions and ask students to use their knowledge to find solutions for themselves. ${ }^{1}$

Freire also suggests students learn by engaging with the world. Clinical exposure is considered the standard, but I propose greater community engagement in the curriculum. ${ }^{1}$ With this approach, students are not passive observers but are active participants. This will make learning relevant and important to the lives of dental students.

This article aims to challenge the dental education system and open a dialogue on how dental students can become critical thinkers in a world where technology and demographics are changing rapidly due to globalisation.

\section{References}

1. Freire P. Pedagogy of the oppressed. Print book: English ed. New York: Herder and Herder; 1970.

2. Drew C. Helpful Professor. 2021. Available online at: https://helpfulprofessor.com/banking-model/ (Accessed February 2021).

\section{SMALL BUT MASSIVE}

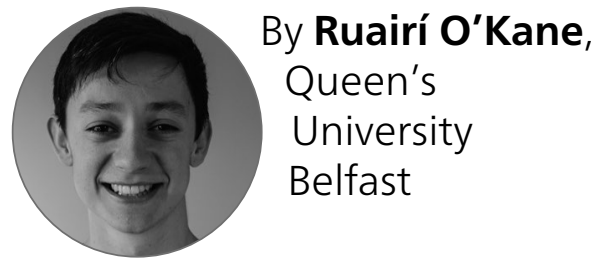

If you've been on student clinics, you have likely written 'OHI' and 'Diet Advice' when devising treatment plans for patients. If you haven't started clinics yet, 'OHI' stands for Oral Hygiene Instruction. Whilst we might be aware of the importance of these two aspects in oral health, do we spend as much time considering how we achieve this as we do when learning other parts of dentistry?

One of the things that challenge us when delivering $\mathrm{OHI}$ and diet advice is recognising that achieving complete behaviour change in a high caries risk patient is unlikely to happen overnight. SIGN 138 pointed out that evidence is currently lacking for the effectiveness of interventions on directly preventing dental caries. ${ }^{1}$ Does this mean that our best efforts are in vain, and patients just won't listen to us? Is giving $\mathrm{OHI}$ and diet advice analogous to the futility of Sisyphus, who was forever doomed to pushing a boulder up a hill, only for it to roll back down again?

Thankfully, there is emerging evidence of the effect motivational interviewing and forming action plans can have on improving dental and general health. A randomised controlled trial highlighted the positive impact of an 'if-then plan' where individuals specified what they're going to do, when they'll do it and where they'll do it. This intervention was timed to take 1.16 